\title{
ON A GENERALIZATION \\ OF THE JORDAN CANONICAL FORM THEOREM ON SEPARABLE HILBERT SPACES
}

\author{
RUI SHI \\ (Communicated by Richard Rochberg)
}

\begin{abstract}
We prove a generalization of the Jordan canonical form theorem for a class of bounded linear operators on complex separable Hilbert spaces.
\end{abstract}

\section{INTRODUCTION}

Throughout this paper, all Hilbert spaces discussed are complex and separable while all operators are bounded and linear on the Hilbert spaces. Let $\mathscr{H}$ be a Hilbert space and let $\mathscr{L}(\mathscr{H})$ be the set of bounded linear operators on $\mathscr{H}$. An idempotent $P$ is an operator such that $P^{2}=P$. A projection $Q$ is an idempotent such that $Q=Q^{*}$. Unless otherwise stated, the term algebra will always refer to a unital subalgebra of $\mathscr{L}(\mathscr{H})$ which is closed in the strong operator topology. An operator $A$ on $\mathscr{H}$ is said to be irreducible if its commutant $\{A\}^{\prime} \equiv\{B \in \mathscr{L}(\mathscr{H})$ : $A B=B A\}$ contains no projections other than 0 and $I$, introduced by P. Halmos 11. (The separability assumption is necessary because on a nonseparable Hilbert space every operator is reducible.) An operator $A$ on $\mathscr{H}$ is said to be strongly irreducible if $X A X^{-1}$ is irreducible for every invertible operator $X$ in $\mathscr{L}(\mathscr{H})$, introduced by F. Gilfeather 10. Equivalently, an operator $A$ is strongly irreducible if and only if $\{A\}^{\prime}$ contains no idempotents other than 0 and $I$.

In [18, strongly irreducible operators are considered as analogues of Jordan blocks on complex separable Hilbert spaces. In the past two decades, D. A. Herrero, Yasuo Watatani, Zejian Jiang, C. K. Fong, Chunlan Jiang, Zongyao Wang, Peiyuan $\mathrm{Wu}$, Youqing Ji, Junsheng Fang and many other mathematicians did a lot of work around this class of operators [4, 17, 8, 9, 12, 13, 14, 16, 17.

On a finite-dimensional Hilbert space $\mathscr{K}$, the Jordan canonical form theorem shows that every operator can be uniquely written as a (Banach) direct sum of Jordan blocks up to similarity. This means that for an operator $B$ on $\mathscr{K}$, there is a bounded maximal abelian set of idempotents $\mathscr{Q}$ in $\{B\}^{\prime}$ and $\mathscr{Q}$ is unique up to similarity in $\{B\}^{\prime}$. However, to represent certain operators on $\mathscr{H}$, direct sums of Jordan blocks need to be replaced by direct integrals of strongly irreducible operators with regular Borel measures. In [15, we proved that an operator $A$ on $\mathscr{H}$ is similar to a direct integral of strongly irreducible operators if and only if its

Received by the editors October 27, 2010 and, in revised form, December 18, 2010.

2010 Mathematics Subject Classification. Primary 47A65, 47A67; Secondary 47A15, 47C15.

Key words and phrases. Strongly irreducible operator, masa, direct integral.

The author was supported in part by NSFC Grant \#10731020 and NSFC Grant \#10901046. 
commutant $\{A\}^{\prime}$ contains a bounded maximal abelian set of idempotents. Related concepts about direct integrals can be found in [3, 5, 6, 19, 20,

For an operator $A$ on $\mathscr{H}$, a direct integral decomposition of $A$ is said to be a strongly irreducible decomposition (S. I. D.) of $A$ if the integrand is strongly irreducible almost everywhere on the domain of integration. An S. I. D. of $A$ is said to be unique up to similarity if for bounded maximal abelian sets of idempotents $\mathscr{P}$ and $\mathscr{Q}$ in $\{A\}^{\prime}$, there is an invertible operator $X$ in $\{A\}^{\prime}$ such that $X \mathscr{P} X^{-1}=\mathscr{Q}$.

In this paper, we study when $A$ has a unique S. I. D. up to similarity, for $A$ similar to a direct integral of strongly irreducible operators.

\section{UPPER TRIANGULAR REPRESENTATION AND MAIN THEOREMS}

If an operator $A$ in $\mathscr{L}(\mathscr{H})$ is similar to a direct integral of strongly irreducible operators, then there is an invertible operator $X$ in $\mathscr{L}(\mathscr{H})$ such that $X A X^{-1}$ has an S. I. D. in the form

$$
X A X^{-1}=\left(\bigoplus_{n=1}^{\infty} \int_{\Lambda_{n}}\left(X A X^{-1}\right)(\lambda) d \mu(\lambda)\right) \oplus \int_{\Lambda_{\infty}}\left(X A X^{-1}\right)(\lambda) d \mu(\lambda) .
$$

Here $\mu$ is a regular Borel measure. Write $\Lambda$ for $\bigcup_{n=1}^{n=\infty} \Lambda_{n}$. The sets $\Lambda_{\infty}$ and $\Lambda_{n}$ for $n$ in $\mathbb{N}$ are bounded Borel and pairwise disjoint. For $n$ in $\mathbb{N}$ and almost every $\lambda$ in $\Lambda_{n}$, the dimension of the fibre space $\mathscr{H}_{\lambda}$ is $n$. For almost every $\lambda$ in $\Lambda_{\infty}$, the dimension of the fibre space $\mathscr{H}_{\lambda}$ is $\infty$. (For fibre spaces, see [1, §2.) Some $\Lambda_{n}$ s and $\Lambda_{\infty}$ may be of measure zero. The partitioned measure space corresponding to the S. I. D. of $X A X^{-1}$ is denoted by $\left\{\Lambda, \mu,\left\{\Lambda_{n}\right\}_{n=1}^{n=\infty}\right\}$.

For a nonzero normal operator $N$ on $\mathscr{H}$, the tensor product $I \otimes N$ does not have unique S. I. D. up to similarity, where $I$ is the identity operator on $\mathscr{H}$ and $\operatorname{dim} \mathscr{H}=\infty$. If $A$ is similar to a normal operator $N$, then the $\mathrm{S}$. I. D. of $A$ is unique up to similarity if and only if the multiplicity function $m_{N}$ for $N$ is bounded a.e. on $\sigma(N)$. Based on this, we can construct a nonnormal operator which does not have a unique S. I. D. up to similarity if $\mu\left(\Lambda_{\infty}\right) \neq 0$ in (1). If $\mu\left(\Lambda_{\infty}\right)=0$, then the S. I. D. of $X A X^{-1}$ is of the form

$$
X A X^{-1}=\bigoplus_{n=1}^{\infty} \int_{\Lambda_{n}}\left(X A X^{-1}\right)(\lambda) d \mu(\lambda) .
$$

By ([2, Corollary 2), there is a unitary operator $U$ such that the equation

$$
U\left(X A X^{-1}\right) U^{*}(\lambda)=U(\lambda)\left(X A X^{-1}\right)(\lambda) U^{*}(\lambda)
$$

holds a.e. on $\Lambda$ and $U\left(X A X^{-1}\right) U^{*}(\lambda)$ is upper triangular in $M_{n}(\mathbb{C})$ for $\lambda$ a.e. in $\Lambda_{n}$. Write $\mu_{n}$ for $\left.\mu\right|_{\Lambda_{n}}, 1 \leq n<\infty$. Without loss of generality, we assume that

$$
X A X^{-1}=\bigoplus_{n=1}^{\infty} \int_{\Lambda_{n}}\left(\begin{array}{ccccc}
M_{\phi_{n}} & M_{\phi_{12}^{n}} & M_{\phi_{13}^{n}} & \cdots & M_{\phi_{1 n}^{n}} \\
0 & M_{\phi_{n}} & M_{\phi_{23}^{n}} & \cdots & M_{\phi_{2 n}^{n}} \\
0 & 0 & M_{\phi_{n}} & \cdots & M_{\phi_{3 n}^{n}} \\
\vdots & \vdots & \vdots & \ddots & \vdots \\
0 & 0 & 0 & \cdots & M_{\phi_{n}}
\end{array}\right)_{n \times n}(\lambda) d \mu(\lambda),
$$

where $\phi_{n}, \phi_{i j}^{n} \in L^{\infty}\left(\mu_{n}\right)$, and $M_{\phi_{n}}$ and $M_{\phi_{i j}^{n}}$ are multiplication operators. The scalar-valued spectral measure for $M_{\phi_{n}}$ is $\nu_{n} \equiv \mu_{n} \circ \phi_{n}^{-1}$. Let the set $\left\{\Gamma_{n m}\right\}_{m=1}^{m=\infty}$ be a Borel partition of $\sigma\left(M_{\phi_{n}}\right)$ corresponding to the multiplicity function $m_{\phi_{n}}$ for 
$M_{\phi_{n}}$ on $\sigma\left(M_{\phi_{n}}\right)$, where $m_{\phi_{n}}(\lambda)=m, \forall \lambda \in \Gamma_{n m}$. Write $\nu_{n m}$ for $\left.\nu_{n}\right|_{\Gamma_{n m}}, 1 \leq m \leq \infty$. We find that the functions $m_{\phi_{n}}$ play a significant role in studying the uniqueness of an S. I. D. of $A$ up to similarity. (Note that $\phi_{i j}^{n}$ does not stand for $\phi_{i j}$ to the power of $n$ here. The symbol $n$ is only a superscript.)

Definition 2.1. The function $m_{\phi_{n}}$ on $\sigma\left(M_{\phi_{n}}\right)$ is said to be the characteristic function of $S$. I. D. of $X A X^{-1}$ corresponding to $\Lambda_{n}$.

In the rest of this paper, we write the partitioned measure space corresponding to the S. I. D. of $X A X^{-1}$ as $\left\{\Lambda, \mu,\left\{\Lambda_{n}, m_{\phi_{n}}\right\}_{n=1}^{\infty}\right\}$.

The purpose of this paper is to give a sufficient condition such that the S. I. D. of $A$ in (2) is unique up to similarity. Precisely we prove the following theorems.

Theorem 2.2. For a fixed $n$, assume that $T \in \mathscr{L}(\mathscr{H})$ is a direct integral of upper triangular strongly irreducible operators and the corresponding measure space is $\left\{\Lambda_{n}, \mu_{n},\left\{\Lambda_{n}, m_{\phi_{n}}\right\}\right\}$ as in (3). If there is a unitary operator $U$ such that both

$$
U M_{\phi_{n}} U^{*}=M_{z_{\infty}}^{(\infty)} \oplus M_{z_{1}} \oplus M_{z_{2}}^{(2)} \oplus \cdots
$$

and

$$
U M_{\phi_{i j}^{n}} U^{*}=M_{\psi_{\infty}^{n, i j}}^{(\infty)} \oplus M_{\psi_{1}^{n, i j}} \oplus M_{\psi_{2}^{n, i j}}^{(2)} \oplus \cdots
$$

hold, where $\psi_{m}^{n, i j}$ and $z_{m}$ are in $L^{\infty}\left(\nu_{n m}\right)$, and $z_{m}(t)=t, \forall t \in \Gamma_{n m}$, then the following statements are equivalent:

(1) The bounded $\nu_{n}$-measurable multiplicity function $m_{\phi_{n}}$ is simple on $\sigma\left(M_{\phi_{n}}\right)$.

(2) The S. I. D. of T is unique up to similarity.

(3) The $K_{0}$ group of $\{T\}^{\prime}$ is of the form

$$
K_{0}\left(\{T\}^{\prime}\right) \cong\left\{f: \sigma\left(M_{\phi_{n}}\right) \rightarrow \mathbb{Z} \mid f \text { is bounded Borel }\right\} .
$$

The condition in this theorem is significant and reasonable. We show this in the proofs in $\S 3$. The following theorem is a generalized version of the above theorem.

Theorem 2.3. Assume that $T \in \mathscr{L}(\mathscr{H})$ is a direct integral of upper triangular strongly irreducible operators and the corresponding partitioned measure space is $\left\{\Lambda, \mu,\left\{\Lambda_{n}, m_{\phi_{n}}\right\}_{n=1}^{\infty}\right\}$ as in (3). The set $\Lambda_{n}$ is of $\mu$-measure 0 for all but finitely many $n$ in $\mathbb{N}$. If the spectral measures for $\left\{M_{\phi_{n}}\right\}_{n=1}^{\infty}$ are mutually singular and there is a unitary operator $U \in\{T\}^{\prime}$ satisfying the condition in Theorem 2.2 w.r.t. $\phi_{n}$ and $\phi_{i j}^{n}(i>j)$ on every $\Lambda_{n}$, then the following statements are equivalent:

(1) The bounded $\nu_{n}$-measurable multiplicity function $m_{\phi_{n}}$ is simple on $\sigma\left(M_{\phi_{n}}\right)$ for $n$ in $\mathbb{N}$.

(2) The S. I. D. of T is unique up to similarity.

(3) The $K_{0}$ group of $\{T\}^{\prime}$ is of the form

$$
K_{0}\left(\{T\}^{\prime}\right) \cong\left\{f: \bigcup_{n=1}^{\infty} \sigma\left(M_{\phi_{n}}\right) \rightarrow \mathbb{Z} \mid f \text { is bounded Borel }\right\} .
$$

The rest of this paper is organized as follows. In Section 3, first we prove a special case of Theorem 3.3 in Lemma 3.2 and then we prove Theorem 3.3 in three lemmas. Corollary 3.7 is to characterize the $K_{0}$ group of the commutant of the operator $T$ in Theorem 3.3. In Example 3.8, we construct an operator and compute the corresponding $K_{0}$ group. Finally, we prove Theorem 2.2 and Theorem 2.3. The operator $T$ in Theorem 3.3 indicates why we add a condition about the unitary operator in Theorem 2.2 . 


\section{ProOFS}

The following lemma reveals an important property which is applied in other lemmas in this paper.

Lemma 3.1. Assume that $\phi, \phi_{i j} \in L^{\infty}\left(\mu_{n}\right)$, where $1 \leq i, j \leq n$ and $\Lambda_{n}$ is as in (1). The multiplication operators $M_{\phi}$ and $M_{\phi_{i j}}$ are on $L^{2}\left(\mu_{n}\right)$. Then the upper triangular form

$$
T=\left(\begin{array}{ccccc}
M_{\phi} & M_{\phi_{12}} & M_{\phi_{13}} & \cdots & M_{\phi_{1 n}} \\
0 & M_{\phi} & M_{\phi_{23}} & \cdots & M_{\phi_{2 n}} \\
0 & 0 & M_{\phi} & \cdots & M_{\phi_{3 n}} \\
\vdots & \vdots & \vdots & \ddots & \vdots \\
0 & 0 & 0 & \cdots & M_{\phi}
\end{array}\right)_{n \times n}
$$

is strongly irreducible a.e. on $\Lambda_{n}$ if and only if $\phi_{i, i+1}(\lambda) \neq 0$ holds a.e. on $\Lambda_{n}$ for $i=1,2, \ldots, n-1$. The underlying Hilbert space of $T$ is denoted by $\mathscr{H}$.

Proof. For $\lambda$ in $\Lambda_{n}$, if $T(\lambda)$ is strongly irreducible in $M_{n}(\mathbb{C})$, then there is an invertible operator $X$ in $M_{n}(\mathbb{C})$ such that $T(\lambda) X=X J_{\sigma(T(\lambda))}$, where $J_{\sigma(T(\lambda))}$ is a Jordan block in $M_{n}(\mathbb{C})$ with spectrum $\sigma(T(\lambda))$. The equation $T(\lambda) X=X J_{\sigma(T(\lambda))}$ becomes

$$
\begin{gathered}
\left(\begin{array}{ccccc}
\alpha & \alpha_{12} & \alpha_{13} & \cdots & \alpha_{1 n} \\
0 & \alpha & \alpha_{23} & \cdots & \alpha_{2 n} \\
0 & 0 & \alpha & \cdots & \alpha_{3 n} \\
\vdots & \vdots & \vdots & \ddots & \vdots \\
0 & 0 & 0 & \cdots & \alpha
\end{array}\right)\left(\begin{array}{ccccc}
x_{11} & x_{12} & x_{13} & \cdots & x_{1 n} \\
x_{21} & x_{22} & x_{23} & \cdots & x_{2 n} \\
x_{31} & x_{32} & x_{33} & \cdots & x_{3 n} \\
\vdots & \vdots & \vdots & \ddots & \vdots \\
x_{n 1} & x_{n 2} & x_{n 3} & \cdots & x_{n n}
\end{array}\right) \\
=\left(\begin{array}{ccccc}
x_{11} & x_{12} & x_{13} & \cdots & x_{1 n} \\
x_{21} & x_{22} & x_{23} & \cdots & x_{2 n} \\
x_{31} & x_{32} & x_{33} & \cdots & x_{3 n} \\
\vdots & \vdots & \vdots & \ddots & \vdots \\
x_{n 1} & x_{n 2} & x_{n 3} & \cdots & x_{n n}
\end{array}\right)\left(\begin{array}{ccccc}
\alpha & 1 & 0 & \cdots & 0 \\
0 & \alpha & 1 & \cdots & 0 \\
0 & 0 & \alpha & \cdots & 0 \\
\vdots & \vdots & \vdots & \ddots & \vdots \\
0 & 0 & 0 & \cdots & \alpha
\end{array}\right),
\end{gathered}
$$

where $\alpha=\sigma(T(\lambda))$. This equation implies that $x_{i j}=0$ for $i>j$ and $\alpha_{i-1, i} x_{i i}=$ $x_{i-1, i-1}$ for $i=2,3, \ldots, n$. Therefore the invertibility of $X$ implies that $\alpha_{i-1, i} \neq 0$ holds for $i=2,3, \ldots, n$.

On the other hand, if $\alpha_{i-1, i} \neq 0$ holds for $i=2,3, \ldots, n$, then every operator $X$ in $M_{n}(\mathbb{C})$ satisfying $T(\lambda) X=X T(\lambda)$ can be expressed in the form

$$
X=\left(\begin{array}{ccccc}
x_{11} & x_{12} & x_{13} & \cdots & x_{1 n} \\
0 & x_{11} & x_{23} & \cdots & x_{2 n} \\
0 & 0 & x_{11} & \cdots & x_{3 n} \\
\vdots & \vdots & \vdots & \ddots & \vdots \\
0 & 0 & 0 & \cdots & x_{11}
\end{array}\right) .
$$

If $X$ is an idempotent, then it must be $I$ or 0 . Thus $T(\lambda)$ is strongly irreducible.

Lemma 3.2. Suppose that an operator $T$ is assumed as in $(4), \phi_{i, i+1}(\lambda) \neq 0$ holds a.e. on $\Lambda_{n}$ for $i=1,2, \ldots, n-1$, and $\phi$ is one to one a.e. on $\Lambda_{n}$. Then the S. I. D. of $T$ is unique up to similarity. 
Proof. Let $E(\cdot)$ be the spectral measure of $M_{\phi}$. Thus $E^{(n)}(\cdot)$ is the spectral measure of $M_{\phi}^{(n)}$. Meanwhile, the spectral measures of $M_{\phi}$ and $M_{z}$ have the same range. First we show that $\{T\}^{\prime} \subseteq\left\{M_{\phi}^{(n)}\right\}^{\prime}$. Equivalently, we need to prove that for every Borel subset $\sigma$ of $\sigma\left(M_{\phi}\right)$ and $X \in\{T\}^{\prime}$, the projection $E^{(n)}(\sigma)$ reduces $X$.

If we write $\mu_{n 1}$ for $\left.\mu_{n}\right|_{\phi^{-1}(\sigma)}$ and $\mu_{n 2}$ for $\left.\mu_{n}\right|_{\Lambda_{n} \backslash \phi^{-1}(\sigma)}$, then we have

$$
\left[\operatorname{ran}\left(E^{(n)}(\sigma)\right)\right]=\left(L^{2}\left(\mu_{n 1}\right)\right)^{(n)},\left[\operatorname{ran}\left(I-E^{(n)}(\sigma)\right)\right]=\left(L^{2}\left(\mu_{n 2}\right)\right)^{(n)} .
$$

The operators $T$ and $X$ can be expressed in the form

$$
T=\left(\begin{array}{cc}
T_{1} & 0 \\
0 & T_{2}
\end{array}\right) \begin{aligned}
& \left(L^{2}\left(\mu_{n 1}\right)\right)^{(n)} \\
& \left(L^{2}\left(\mu_{n 2}\right)\right)^{(n)}
\end{aligned}, \quad X=\left(\begin{array}{cc}
X_{11} & X_{12} \\
X_{21} & X_{22}
\end{array}\right) \begin{aligned}
& \left(L^{2}\left(\mu_{n 1}\right)\right)^{(n)} \\
& \left(L^{2}\left(\mu_{n 2}\right)\right)^{(n)}
\end{aligned}
$$

where

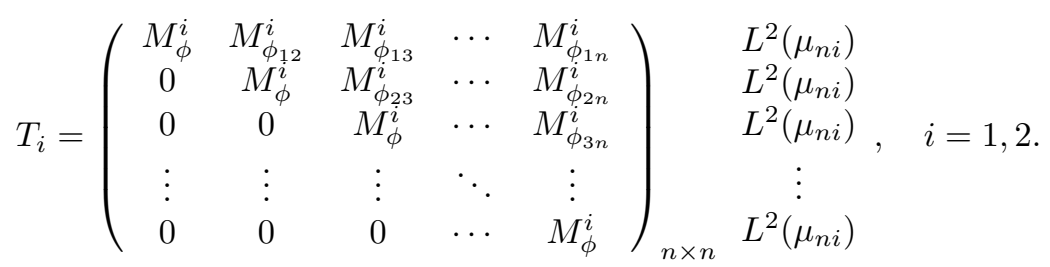

The equation $T_{1} X_{12}=X_{12} T_{2}$ and the fact that $M_{\phi}^{1}$ and $M_{\phi}^{2}$ have mutually singular scalar-valued spectral measures imply that $X_{12}=0$. In the same way we obtain $X_{21}=0$. Therefore $X \in\left\{M_{\phi}^{(n)}\right\}^{\prime}$.

By Lemma 3.1, we compute the equation $T X=X T$ and obtain that the operator $X$ has the form

$$
X=\left(\begin{array}{ccccc}
M_{\psi} & M_{\psi_{12}} & M_{\psi_{13}} & \cdots & M_{\psi_{1 n}} \\
0 & M_{\psi} & M_{\psi_{23}} & \cdots & M_{\psi_{2 n}} \\
0 & 0 & M_{\psi} & \cdots & M_{\psi_{3 n}} \\
\vdots & \vdots & \vdots & \ddots & \vdots \\
0 & 0 & 0 & \cdots & M_{\psi}
\end{array}\right)_{n \times n}
$$

where $\psi, \psi_{i j} \in L^{\infty}\left(\mu_{n}\right)$. Hence every idempotent in $\{T\}^{\prime}$ is a spectral projection of $M_{\phi}^{(n)}$. This means that in the commutant of $T$, there is one and only one bounded maximal abelian set of idempotents.

Theorem 3.3. If an operator $T$ is assumed as in Lemma 3.2 and $m$ is a positive integer, then the S. I. D. of $T^{(m)}$ is unique up to similarity.

We denote by $\mathscr{P}$ the set of projections in $\{T\}^{\prime}$. This is the only maximal abelian set of idempotents in $\{T\}^{\prime}$. The set $\mathscr{P} \oplus \cdots \oplus \mathscr{P}(m$ copies $)$ is a bounded maximal abelian set of idempotents in $M_{m}\left(\{T\}^{\prime}\right)$. We prove Theorem 3.3 in three lemmas.

Lemma 3.4. If $Q \in M_{m}\left(\{T\}^{\prime}\right)$ is an idempotent, then there is an invertible operator $X \in M_{m}\left(\{T\}^{\prime}\right)$ such that the operator $X Q X^{-1}$ belongs to the set $\mathscr{P} \oplus \cdots \oplus$ $\mathscr{P}(m$ copies $)$.

Proof. The idempotent $Q$ is decomposable with respect to the diagonal algebra generated by the set $\mathscr{P}^{(m)} \equiv\{P \oplus P \oplus \cdots \oplus P(m$ copies $): P \in \mathscr{P}\}$. The measure space is $\Lambda_{n}$. Based on (5) in Lemma 3.2, the operator $Q$ can be expressed in the 
form

$$
Q=\left(\begin{array}{ccccccc}
M_{\psi^{11}} & \cdots & M_{\psi_{1 n}^{11}} & & M_{\psi^{1 m}} & \cdots & M_{\psi_{1 n}^{1 m}} \\
\vdots & \ddots & \vdots & \cdots & \vdots & \ddots & \vdots \\
0 & \cdots & M_{\psi^{11}} & & 0 & \cdots & M_{\psi^{1 m}} \\
& \vdots & & \ddots & & \vdots & \\
M_{\psi^{m 1}} & \cdots & M_{\psi_{1 n}^{m 1}} & & M_{\psi^{m m}} & \cdots & M_{\psi_{1 n}^{m m}} \\
\vdots & \ddots & \vdots & \cdots & \vdots & \ddots & \vdots \\
0 & \cdots & M_{\psi^{m 1}} & & 0 & \cdots & M_{\psi^{m m}}
\end{array}\right)_{m n \times m n}
$$

There is a unitary operator $U_{1}$ such that the operator $Q_{1}=U_{1} Q U_{1}^{*}$ is represented as a block upper triangular operator-valued matrix in the form

$$
Q_{1}=\left(\begin{array}{ccccc}
Q_{11}^{1} & Q_{12}^{1} & Q_{13}^{1} & \cdots & Q_{1 n}^{1} \\
0 & Q_{11}^{1} & Q_{23}^{1} & \cdots & Q_{2 n}^{1} \\
0 & 0 & Q_{11}^{1} & \cdots & Q_{3 n}^{1} \\
\vdots & \vdots & \vdots & \ddots & \vdots \\
0 & 0 & 0 & \cdots & Q_{11}^{1}
\end{array}\right)_{n \times n},
$$

where

$$
Q_{11}^{1}=\left(\begin{array}{ccc}
M_{\psi^{11}} & \cdots & M_{\psi^{1 m}} \\
\vdots & \ddots & \vdots \\
M_{\psi^{m 1}} & \cdots & M_{\psi^{m m}}
\end{array}\right)_{m \times m} \quad, \quad Q_{i j}^{1}=\left(\begin{array}{ccc}
M_{\psi_{i j}^{11}} & \cdots & M_{\psi_{i j}^{1 m}} \\
\vdots & \ddots & \vdots \\
M_{\psi_{i j}^{m 1}} & \cdots & M_{\psi_{i j}^{m m}}
\end{array}\right)_{m \times m},
$$

for $1 \leq i<j \leq n$. Notice that $Q_{11}^{1}$ is an idempotent.

Next, we prove that there is an invertible operator $X_{2}$ in $M_{m}\left(L^{\infty}\left(\mu_{n}\right)\right)$ such that $X_{2} Q_{11}^{1} X_{2}^{-1}$ is a projection in the form

$$
X_{2} Q_{11}^{1} X_{2}^{-1}=\left(\begin{array}{cccc}
M_{\chi_{S_{1}}} & 0 & \cdots & 0 \\
0 & M_{\chi_{S_{2}}} & \cdots & 0 \\
\vdots & \vdots & \ddots & \vdots \\
0 & 0 & \cdots & M_{\chi_{S_{m}}}
\end{array}\right)_{m \times m}
$$

where $S_{i}$ is a Borel subset of $\Lambda_{n}$ for $i=1,2, \ldots, m$.

For any positive integer $k$, there is a positive integer $l_{k}$ such that given any idempotent $P$ in $M_{m}(\mathbb{C})$ with norm less than $k$ there is an invertible operator $X$ with norm less than $l_{k}$ such that $X P X^{-1}$ is similar to the corresponding Jordan block. That is because any idempotent in $M_{m}(\mathbb{C})$ is unitarily equivalent to a block matrix in the form

$$
\left(\begin{array}{ll}
I & R \\
0 & 0
\end{array}\right)
$$

and

$$
\left(\begin{array}{cc}
I & R \\
0 & I
\end{array}\right)\left(\begin{array}{ll}
I & R \\
0 & 0
\end{array}\right)\left(\begin{array}{cc}
I & -R \\
0 & I
\end{array}\right)=\left(\begin{array}{ll}
I & 0 \\
0 & 0
\end{array}\right)
$$

For the set defined in ([2], Corollary 3$)$

$$
\begin{array}{r}
\mathscr{E}_{l_{k}}=\left\{(A, J, X) \in M_{m}(\mathbb{C}) \times M_{m}(\mathbb{C}) \times M_{m}(\mathbb{C}): J\right. \text { is in Jordan form, } \\
\left.\|X\| \leq l_{k},\left\|X^{-1}\right\| \leq l_{k} \text { and } X A X^{-1}=J\right\},
\end{array}
$$


the set $\pi_{1}\left(\mathscr{E}_{l_{k}}\right)$ contains every idempotent whose norm is less than $k$. By ([2], Theorem 1), the Borel map $\phi_{l_{k}}: \pi_{1}\left(\mathscr{E}_{l_{k}}\right) \rightarrow \pi_{3}\left(\mathscr{E}_{l_{k}}\right)$ is bounded. Therefore the equivalence class of $\phi_{l_{\left.\Gamma\left\|Q_{11}^{1}\right\|\right\rceil}} \circ Q_{11}^{1}(\cdot)$ is the $X_{2}$ we need in $M_{m}\left(L^{\infty}\left(\mu_{n}\right)\right)$.

Write $Q_{2}$ for $\left(X_{2}^{(n)}\right) Q_{1}\left(X_{2}^{(n)}\right)^{-1}$. There is a unitary operator $U_{3}$ in $M_{m}\left(L^{\infty}\left(\mu_{n}\right)\right)$ such that $\left(U_{3}^{(n)}\right) Q_{2}\left(U_{3}^{(n)}\right)^{*}$ can be expressed in the form

$$
\left(U_{3}^{(n)}\right) Q_{2}\left(U_{3}^{(n)}\right)^{*}=\left(\begin{array}{ccccc}
Q_{11}^{3} & Q_{12}^{3} & Q_{13}^{3} & \cdots & Q_{1 n}^{3} \\
0 & Q_{11}^{3} & Q_{23}^{3} & \cdots & Q_{2 n}^{3} \\
0 & 0 & Q_{11}^{3} & \cdots & Q_{3 n}^{3} \\
\vdots & \vdots & \vdots & \ddots & \vdots \\
0 & 0 & 0 & \cdots & Q_{11}^{3}
\end{array}\right)_{n \times n},
$$

where

$$
Q_{11}^{3}=\left(\begin{array}{cccc}
M_{\chi_{S_{1}}} & 0 & \cdots & 0 \\
0 & M_{\chi_{S_{2}}} & \cdots & 0 \\
\vdots & \vdots & \ddots & \vdots \\
0 & 0 & \cdots & M_{\chi_{S_{m}}}
\end{array}\right)_{m \times m}
$$

The set $S_{i}$ is a Borel subset of $\Lambda_{n}$ for $i=1,2, \ldots, m$, and $S_{i+1} \subseteq S_{i}$ for $i=$ $1,2, \ldots, m-1$. Write $Q_{3}$ for $\left(U_{3}^{(n)}\right) Q_{2}\left(U_{3}^{(n)}\right)^{*}$. Notice that $Q_{3}$ belongs to $\left\{U_{1} T^{(m)} U_{1}^{*}\right\}^{\prime}$.

Next, we prove that there is an invertible operator $X_{4}$ in $\left\{U_{1} T^{(m)} U_{1}^{*}\right\}^{\prime}$ such that $X_{4} Q_{3} X_{4}^{-1}$ equals the following projection:

$$
Q_{4}=\left(\begin{array}{ccccc}
Q_{11}^{3} & 0 & 0 & \cdots & 0 \\
0 & Q_{11}^{3} & 0 & \cdots & 0 \\
0 & 0 & Q_{11}^{3} & \cdots & 0 \\
\vdots & \vdots & \vdots & \ddots & \vdots \\
0 & 0 & 0 & \cdots & Q_{11}^{3}
\end{array}\right)_{n \times n}
$$

First, multiply each entry in the lower triangular $m \times m$ matrix form of $Q_{i, i+1}^{3}$ by -1 and denote this new $m \times m$ matrix form by $X_{i, i+1}^{3}$, for $i=1,2, \ldots, n-1$. In $\left\{U_{1} T^{(m)} U_{1}^{*}\right\}^{\prime}$, we can construct an operator $X_{1}^{3}$ in the form

$$
X_{1}^{3}=\left(\begin{array}{ccccc}
I & X_{12}^{3} & *_{13}^{1} & \cdots & *_{1 n}^{1} \\
0 & I & X_{23}^{3} & \cdots & *_{2 n}^{1} \\
0 & 0 & I & \cdots & *_{3 n}^{1} \\
\vdots & \vdots & \vdots & \ddots & \vdots \\
0 & 0 & 0 & \cdots & I
\end{array}\right)_{n \times n}
$$

The operator $X_{1}^{3}$ is invertible and $\sigma\left(X_{1}^{3}\right)=\{1\}$. The fact that $\left\{U_{1} T^{(m)} U_{1}^{*}\right\}^{\prime}$ is a subalgebra of $\mathscr{L}\left(\mathscr{H}^{(m)}\right)$ implies that the equation $\sigma\left(X_{1}^{3}\right)=\{1\}$ holds in $\left\{U_{1} T^{(m)} U_{1}^{*}\right\}^{\prime}$. Thus $X_{1}^{3}$ is invertible in $\left\{U_{1} T^{(m)} U_{1}^{*}\right\}^{\prime}$. Therefore the operator 
$\left(X_{1}^{3}\right) Q_{3}\left(X_{1}^{3}\right)^{-1}$ is in the form

$$
\left(\begin{array}{ccccc}
Q_{11}^{3} & 0 & *_{13}^{2} & \cdots & *_{1 n}^{2} \\
0 & Q_{11}^{3} & 0 & \cdots & *_{2 n}^{2} \\
0 & 0 & Q_{11}^{3} & \cdots & *_{3 n}^{2} \\
\vdots & \vdots & \vdots & \ddots & \vdots \\
0 & 0 & 0 & \cdots & Q_{11}^{3}
\end{array}\right)_{n \times n} .
$$

Repeat the above procedure. We construct invertible operators $X_{i}^{3}$ one by one in $\left\{U_{1} T^{(m)} U_{1}^{*}\right\}^{\prime}$, for $i=1, \ldots, n-1$. After $n-1$ steps, we obtain $Q_{4}$. Denote by $X_{4}$ the product of the $X_{i}^{3} \mathrm{~s}$. The equation $Q_{4}=X_{4} Q_{3} X_{4}^{-1}$ holds. Therefore $X=U_{1}^{*} X_{4} U_{3}^{(n)} X_{2}^{(n)} U_{1}$ is the invertible operator in $M_{m}\left(\{T\}^{\prime}\right)$ such that $X Q X^{-1}$ is a projection in $\mathscr{P} \oplus \cdots \oplus \mathscr{P}(m$ copies $)$.

Lemma 3.5. If $\mathscr{Q}$ is a bounded maximal abelian set of idempotents in $M_{m}\left(\{T\}^{\prime}\right)$, then there is a subset $\left\{Q_{i}\right\}_{i=1}^{2^{m}} \subseteq \mathscr{Q}$ such that for $\lambda$ in $\Lambda_{n}$, the equation $\left\{Q_{i}(\lambda)\right\}_{i=1}^{2^{m}}=$ $\mathscr{Q}(\lambda)$ holds a.e. on $\Lambda_{n}$.

Proof. By the above lemma, we know that for every $Q$ in $\mathscr{Q}$, there is an invertible operator $X$ in $M_{m}\left(\{T\}^{\prime}\right)$ such that $X Q X^{-1}$ is a projection in $\mathscr{P} \oplus \cdots \oplus \mathscr{P}(m$ copies $)$. Thus we define a function

$$
r_{Q}(\lambda)=\frac{1}{n} \operatorname{rank}(Q(\lambda)), \forall Q \in \mathscr{Q}, \lambda \in \Lambda_{n} .
$$

The function $r_{Q}$ is in the equivalence class of certain simple functions. To prove this lemma, we only need to show that there are $m$ idempotents $Q_{i}$ in $\mathscr{Q}$ such that the equation $r_{Q_{i}}(\lambda)=1$, for $i=1, \ldots, m$, holds a.e. on $\Lambda_{n}$ and $Q_{i} Q_{j}=0$, for $i \neq j$. We prove this in two steps.

Step 1. We prove that there is an idempotent $Q^{\prime}$ in $\mathscr{Q}$ such that the relation $0<r_{Q^{\prime}}(\lambda)<m$ holds a.e. on $\Lambda_{n}$.

If the relation $\left\{r_{Q}(\lambda): Q \in \mathscr{Q}\right\}=\{0, m\}$ holds a.e. on $\Lambda_{n}$, then we can construct a strongly measurable operator-valued constant function $Q^{\prime}(\cdot)$ satisfying the following properties:

(1) $Q^{\prime}(\cdot)$ is nontrivial a.e. on $\Lambda_{n}$.

(2) The equivalence class $Q^{\prime}$ of $Q^{\prime}(\cdot)$ is a projection in $M_{m}\left(\{T\}^{\prime}\right)$ commuting with every idempotent in $\mathscr{Q}$.

(3) $Q^{\prime}$ does not belong to $\mathscr{Q}$.

This contradicts the assumption that $\mathscr{Q}$ is a maximal abelian set of idempotents. Therefore, there are an idempotent $Q_{1}^{\prime}$ in $\mathscr{Q}$ and a Borel subset $\Lambda_{n 1}$ of $\Lambda_{n}$ with nonzero measure such that the relation $0<r_{Q_{1}^{\prime}}(\lambda)<m$ holds a.e. on $\Lambda_{n 1}$. Thus there are an idempotent $Q_{2}^{\prime}$ in $\mathscr{Q}$ and a Borel subset $\Lambda_{n 2}$ of $\Lambda_{n} \backslash \Lambda_{n 1}$ with nonzero measure such that the relation $0<r_{Q_{2}^{\prime}}(\lambda)<m$ holds a.e. on $\Lambda_{n 2}$. Carrying out this procedure, we obtain a subset $\left\{Q_{i}^{\prime}\right\}_{i=1}^{\infty}$ of $\mathscr{Q}$ and $\Lambda_{n}=\bigcup_{i=1}^{\infty} \Lambda_{n i}$. Define

$$
Q^{\prime}=\sum_{i=1}^{\infty}\left[\chi_{\Lambda_{n i}}\right] \cdot Q_{i}^{\prime}, \quad \chi_{\Lambda_{n i}}(\lambda)= \begin{cases}I \in M_{m n}(\mathbb{C}), & \lambda \in \Lambda_{n i} ; \\ 0 \in M_{m n}(\mathbb{C}), & \lambda \in \Lambda_{n} \backslash \Lambda_{n i} .\end{cases}
$$

The idempotent $Q^{\prime}$ is what we want in step 1 .

Note that $r_{Q^{\prime}}$ is in the equivalence class of a simple function. We can write $\Lambda_{n}$ in the form of a union of disjoint Borel subsets $\Lambda_{n i}^{\prime}$ of $\Lambda_{n}$ such that the equation 
$r_{Q^{\prime}}(\lambda)=i$ holds a.e. on $\Lambda_{n i}^{\prime}$. (Some $\Lambda_{n i}^{\prime}$ s may be of measure zero.) Write

$$
\mathscr{Q}_{i}=\left\{\left[\chi_{\Lambda_{n i}^{\prime}}\right] Q Q^{\prime}: Q \in \mathscr{Q}\right\} \text {, for } i=1, \ldots, m-1 \text {. }
$$

Step 2. We prove that for a fixed $i$ larger than 1 , if the set $\Lambda_{n i}^{\prime}$ is not of measure zero, then there is an idempotent $Q^{\prime \prime} \in \mathscr{Q}_{i}$ such that the relation $0<Q^{\prime \prime}(\lambda)<i$ holds a.e. on $\Lambda_{n i}^{\prime}$.

Suppose that the relation $\left\{r_{Q}(\lambda): Q \in \mathscr{Q}_{i}\right\}=\{0, i\}$ holds a.e. on $\Lambda_{n i}^{\prime}$. By the above lemma we know that there is an invertible operator $X \in M_{m}\left(\{T\}^{\prime}\right)$ such that the equation

$$
X Q^{\prime} X^{-1}(\lambda)=\left(\begin{array}{ccccc}
I & 0 & \cdots & 0 & 0 \\
0 & 0 & & 0 & 0 \\
\vdots & & \ddots & \vdots & \\
0 & 0 & \cdots & I & 0 \\
0 & 0 & & 0 & 0
\end{array}\right)_{2 n \times 2 n} \begin{gathered}
\mathbb{C}^{(i)} \\
\mathbb{C}^{(m-i)} \\
\vdots \\
\mathbb{C}^{(m-i)}
\end{gathered}
$$

holds a.e. on $\Lambda_{n i}^{\prime}$. Therefore we can construct an idempotent $Q^{\prime \prime}$ in $M_{m}\left(\{T\}^{\prime}\right)$ satisfying that:

(1) $Q^{\prime \prime}(\lambda)$ is a proper subidempotent of $Q^{\prime}(\lambda)$ a.e. on $\Lambda_{n i}^{\prime}$.

(2) $Q^{\prime \prime}$ commutes with every idempotent in $\mathscr{Q}_{i}$.

(3) $Q^{\prime \prime}$ does not belong to $\mathscr{Q}_{i}$.

Thus $\mathscr{Q}$ is not a maximal abelian set of idempotents. This is a contradiction. Therefore there are an idempotent $Q_{1}^{\prime \prime} \in \mathscr{Q}_{i}$ and a Borel subset $\Lambda_{n i 1}^{\prime}$ of $\Lambda_{n i}^{\prime}$ with nonzero measure such that the relation $0<r_{Q_{1}^{\prime \prime}}(\lambda)<i$ holds a.e. on $\Lambda_{n i 1}^{\prime}$. Thus there are an idempotent $Q_{2}^{\prime \prime} \in \mathscr{Q}_{i}$ and a Borel subset $\Lambda_{n i 2}^{\prime}$ of $\Lambda_{n i}^{\prime} \backslash \Lambda_{n i 1}^{\prime}$ with nonzero measure such that the relation $0<r_{Q_{2}^{\prime \prime}}(\lambda)<i$ holds a.e. on $\Lambda_{n i 2}^{\prime}$. Carrying out this procedure, we obtain a subset $\left\{Q_{k}^{\prime \prime}\right\}_{k=1}^{\infty}$ of $\mathscr{Q}_{i}$ and $\Lambda_{n i}^{\prime}=\bigcup_{k=1}^{\infty} \Lambda_{n i k}^{\prime}$. Write

$$
Q^{\prime \prime}=\sum_{k=1}^{\infty}\left[\chi_{\Lambda_{n i k}^{\prime}}\right] \cdot Q_{k}^{\prime \prime} .
$$

This idempotent $Q^{\prime \prime}$ is what we want in step 2 .

After finite steps, we obtain that there is an idempotent $Q$ in $\mathscr{Q}$ such that the equation $r_{Q}(\lambda)=1$ holds a.e. on $\Lambda_{n}$.

Repeating the above procedure, we can find $m$ idempotents $Q_{i}$ in $\mathscr{Q}$ such that the equation $r_{Q_{i}}(\lambda)=1$, for $i=1, \ldots, m$, holds a.e. on $\Lambda_{n}$ and $Q_{i} Q_{j}=0$, for $i \neq j$. Thus we can obtain $2^{m}$ idempotents that we need.

Lemma 3.6. If $\mathscr{Q}$ is a bounded maximal abelian set of idempotents in $M_{m}\left(\{T\}^{\prime}\right)$, then there is an invertible operator $X \in M_{m}\left(\{T\}^{\prime}\right)$ such that

$$
X \mathscr{Q} X^{-1}=\mathscr{P} \oplus \cdots \oplus \mathscr{P}(m \text { copies }) .
$$

Proof. By the above lemma, we can find $m$ idempotents $Q_{i}$ in $\mathscr{Q}$ such that the equation $r_{Q_{i}}(\lambda)=1$, for $i=1, \ldots, m$, holds a.e. on $\Lambda_{n}$ and $Q_{i} Q_{j}=0$, for $i \neq j$. For $Q_{1}$, there is an invertible operator $X_{1} \in M_{m}\left(\{T\}^{\prime}\right)$ such that $X_{1} Q_{1} X_{1}^{-1}$ is a projection in $\mathscr{P} \oplus \cdots \oplus \mathscr{P}(m$ copies $)$. The invertible operator $X_{1}$ can be chosen 
such that $X_{1} Q_{1} X_{1}^{-1}$ is in the form

$$
X_{1} Q_{1} X_{1}^{-1}=\left(\begin{array}{cccc}
I & 0 & \cdots & 0 \\
0 & 0 & \cdots & 0 \\
\vdots & \vdots & \ddots & \vdots \\
0 & 0 & \cdots & 0
\end{array}\right)_{m \times m}
$$

Thus there is an invertible operator $X_{2} \in M_{m}\left(\{T\}^{\prime}\right)$ such that $X_{2} X_{1} Q_{1} X_{1}^{-1} X_{2}^{-1}$ and $X_{2} X_{1} Q_{2} X_{1}^{-1} X_{2}^{-1}$ are in the form

$$
\begin{aligned}
& X_{2} X_{1} Q_{1} X_{1}^{-1} X_{2}^{-1}=\left(\begin{array}{cccc}
I & 0 & \cdots & 0 \\
0 & 0 & \cdots & 0 \\
\vdots & \vdots & \ddots & \vdots \\
0 & 0 & \cdots & 0
\end{array}\right)_{m \times m}, \\
& X_{2} X_{1} Q_{2} X_{1}^{-1} X_{2}^{-1}=\left(\begin{array}{cccc}
0 & 0 & \cdots & 0 \\
0 & I & \cdots & 0 \\
\vdots & \vdots & \ddots & \vdots \\
0 & 0 & \cdots & 0
\end{array}\right)_{m \times m} .
\end{aligned}
$$

By this procedure, we obtain $\left\{X_{i}\right\}_{i=1}^{m}$ such that $X Q_{i} X^{-1}$ is in the form

$$
X Q_{i} X^{-1}=\left(\begin{array}{ccccc}
0 & \cdots & 0 & \cdots & 0 \\
\vdots & \ddots & \vdots & \ddots & \vdots \\
0 & \cdots & I & \cdots & 0 \\
\vdots & \ddots & \vdots & \ddots & \vdots \\
0 & \cdots & 0 & \cdots & 0
\end{array}\right)_{m \times m} \quad \vdots \text { th }
$$

where $X=X_{m} X_{m-1} \cdots X_{1}$. This $X$ in $M_{m}\left(\{T\}^{\prime}\right)$ is the invertible operator that we need.

With the above three lemmas, we finish the proof of Theorem 3.3.

Corollary 3.7. If an operator $T$ is assumed as in Theorem 3.3 , then the $K_{0}$ group of $\{T\}^{\prime}$ is isomorphic to the set

$$
K_{0}\left(\{T\}^{\prime}\right) \cong\left\{f: \sigma\left(M_{\phi}\right) \rightarrow \mathbb{Z} \mid f \text { is bounded Borel }\right\} .
$$

We give an example to show that the $K_{0}$ group of the commutant of an operator $T$ as in the above corollary is isomorphic to the corresponding set as (7).

Example 3.8. Let $z(t)=t, t \in[0,1]$. The multiplication operator $M_{z}$ corresponding to $z$ is the operator on $L^{2}([0,1])$ defined by

$$
\left(M_{z} f\right)(t)=t \cdot f(t), \quad f \in L^{2}([0,1]) .
$$

Denote by $T$ the $2 \times 2$ operator-valued matrix in the form

$$
T=\left(\begin{array}{cc}
M_{z} & M_{\psi} \\
0 & M_{z}
\end{array}\right), \psi \in L^{\infty}([0,1]), \psi(\lambda) \neq 0 \text { a.e. on }[0,1] .
$$

By Lemma 3.2, we know that every idempotent $P$ in $\{T\}^{\prime}$ is of the form

$$
P=\left(\begin{array}{cc}
M_{\chi_{S}} & 0 \\
0 & M_{\chi_{S}}
\end{array}\right),
$$


where $S$ is a Borel subset of $[0,1]$. By Theorem 3.3, we know that, for any positive integer $m$, in $\left\{T^{(m)}\right\}^{\prime}$ every idempotent is similar to a projection in the form

$$
P=\left(\begin{array}{cccc}
M_{\chi_{S_{1}}}^{(2)} & 0 & \cdots & 0 \\
0 & M_{\chi_{S_{2}}}^{(2)} & \cdots & 0 \\
\vdots & \vdots & \ddots & \vdots \\
0 & 0 & \cdots & M_{\chi_{S_{m}}}^{(2)}
\end{array}\right)
$$

where $S_{i}$ is a Borel subset of $[0,1]$. Denote the standard trace on $M_{2 m}(\mathbb{C})$ by Tr. The bounded function $\frac{1}{2} \operatorname{Tr}(P(\lambda))$ maps $[0,1]$ in $\mathbb{N}$ for almost every $\lambda$ in $[0,1]$. Therefore, in $\bigcup_{m=1}^{\infty}\left\{T^{(m)}\right\}^{\prime}$, for every idempotent $P$, denote by $[P]$ the class of idempotents similar to $P$. In every $[P]$, there are projections as $(8)$. Define $\rho([P]) \equiv$ $\frac{1}{2} \operatorname{Tr}(P(\cdot))$. It is easy to prove that the set $\left\{[P]: P \in \bigcup_{m=1}^{\infty}\left\{T^{(m)}\right\}^{\prime}\right\}$ is isomorphic to the set $\{f:[0,1] \rightarrow \mathbb{N} \mid f$ is bounded Borel $\}$ and $\rho$ is the isomorphic map. By $K$-theory for Banach algebras, we obtain

$$
K_{0}\left(\{T\}^{\prime}\right) \cong\{f:[0,1] \rightarrow \mathbb{Z} \mid f \text { is bounded Borel }\} .
$$

Lemma 3.9. Suppose that $M_{\phi}$ is the multiplication operator on $L^{2}\left(\mu_{n}\right)$ as in Lemma 3.2. Then for every idempotent $P \in\left\{M_{\phi}^{(\infty)}\right\}^{\prime}$, there is an invertible operator $X$ in $\left\{M_{\phi}^{(\infty)}\right\}^{\prime}$ such that $X P X^{-1}$ is a diagonal projection in $\left\{M_{\phi}^{(\infty)}\right\}^{\prime}$.

Proof. The idempotent $P$ is decomposable on $\left(\Gamma, \mu,\left\{\Gamma_{\infty}\right\}\right), \Gamma=\Lambda_{n}$. For every $\lambda$ in $\Gamma$, the fiber space $\mathscr{H}_{\lambda}$ has dimension $\infty$. First, we construct a bounded Borel set

$$
\begin{array}{r}
\left\{(\lambda, Y, Q) \in \Lambda_{n} \times \mathscr{L}\left(\mathscr{H}_{\lambda}\right) \times \mathscr{L}\left(\mathscr{H}_{\lambda}\right): Y^{-1} P(\lambda) Y=Q,\right. \\
\left.Q \text { is a diagonal projection, }\|Y\| \leq k,\left\|Y^{-1}\right\| \leq k\right\},
\end{array}
$$

where $k$ is a large enough positive integer. By ([3], Proposition 2.1), we obtain an invertible operator $X$ in $\left\{M_{\phi}^{(\infty)}\right\}^{\prime}$ such that $X P X^{-1}$ is a diagonal projection. The operator $X$ is what we need.

Lemma 3.10. If an operator $T \in \mathscr{L}(\mathscr{H})$ is assumed as in Lemma 3.2, then for every idempotent $Q$ in $\left\{T^{(\infty)}\right\}^{\prime}$, there is an invertible operator $X$ in $\left\{T^{(\infty)}\right\}^{\prime}$ such that $X Q X^{-1}$ is in $\mathscr{P} \oplus \cdots \oplus \mathscr{P} \oplus \cdots(\infty$ copies $)$.

By the proofs of Lemma 3.4 and Lemma 3.9, we obtain this lemma.

Proof of Theorem 2.2. By Theorem 3.3 and Corollary 3.7, we obtain (1) $\Rightarrow(2)$ and $(1) \Rightarrow(3)$. When the multiplicity function $m_{\phi_{n}}$ for $M_{\phi_{n}}$ takes $\infty$ on $\sigma\left(M_{\phi_{n}}\right)$, we can construct two bounded maximal abelian sets of idempotents in the commutant of $T$ which are not similar to each other in $\{T\}^{\prime}$. By Lemma 3.10, we know that if $m_{\phi_{n}}$ takes $\infty$ on $\sigma\left(M_{\phi_{n}}\right)$, then the $K_{0}$ group of $\{T(\lambda)\}^{\prime}$ is 0 a.e. on $\Gamma_{n \infty}$.

Proof of Theorem 2.3. Denote by $T_{n}$ the restriction of $T$ acting on $\left(L^{2}\left(\mu_{n}\right)\right)^{(n)}$. The operator $T$ can be expressed as $\bigoplus_{n=1}^{\infty} T_{n}$. Since the spectral measures of $\left\{M_{\phi_{n}}\right\}_{n=1}^{\infty}$ are mutually singular, we obtain

$$
\{T\}^{\prime}=\bigoplus_{n=1}^{\infty}\left\{T_{n}\right\}^{\prime} .
$$

The rest of the proof is essentially an application of Theorem 2.2. 


\section{ACKNOWLEDGMENTS}

The author is grateful to Professor Chunlan Jiang and Professor Guihua Gong for their advice and comments on writing this paper, and to the Department of Mathematics of Hebei Normal University for providing a good research environment.

\section{REFERENCES}

1. M. B. Abrahamse, Multiplication operators. Hilbert space operators (Proc. Conf., Calif. State Univ., Long Beach, Calif., 1977), pp. 17-36, Lecture Notes in Math., 693, Springer, Berlin, 1978. MR0526530 (80b:47042)

2. Edward A. Azoff, Borel measurability in linear algebra. Proc. Amer. Math. Soc. 42 (1974), 346-350. MR0327799 (48:6141)

3. E. Azoff, C. Fong and F. Gilfeather, A reduction theory for non-self-adjoint operator algebras. Trans. Amer. Math. Soc. 224 (1976), 351-366. MR0448109 (56:6419)

4. Y. Cao, J. Fang, and C. Jiang, K-groups of Banach algebras and strongly irreducible decompositions of operators. J. Operator Theory 48 (2) (2002), 235-253. MR1938796 (2004f:47003)

5. John B. Conway, A course in functional analysis. Second edition. Graduate Texts in Mathematics, 96. Springer-Verlag, New York, 1990. MR.1070713 (91e:46001)

6. Kenneth R. Davidson, $C^{*}$-algebras by example (English summary), Fields Institute Monographs, 6. American Mathematical Society, Providence, RI, 1996. MR1402012 (97i:46095)

7. M. Enomoto and Y. Watatani, Relative position of four subspaces in a Hilbert space. Adv. Math. 201 (2) (2006), 263-317. MR2211531(2009d:46044)

8. M. Enomoto and Y. Watatani, Exotic indecomposable systems of four subspaces in a Hilbert space. Integral Equations and Operator Theory 59 (2) (2007), 149-164. MR.2345993 (2009g:47194)

9. M. Enomoto and Y. Watatani, Indecomposable representations of quivers on infinitedimensional Hilbert spaces. J. Funct. Anal. 256 (4) (2009), 959-991. MR2488332 (2010c:47238)

10. F. Gilfeather, Strong reducibility of operators. Indiana Univ. Math. J. 22 (4) (1972), 393-397. MR0303322(46:2460)

11. P. Halmos, Irreducible operators. Michigan Math. J. 15 (1968), 215-223. MR0231233 $(37: 6788)$

12. C. Jiang, Similarity classification of Cowen-Douglas operators. Canad. J. Math. 56 (4) (2004), 742-775. MR2074045(2006d:47037)

13. C. Jiang, X. Guo and K. Ji, K-group and similarity classification of operators. J. Funct. Anal. 225 (1) (2005), 167-192. MR2149922 (2006c:47023)

14. C. Jiang and K. Ji, Similarity classification of holomorphic curves. Adv. Math. 215 (2) (2007), 446-468. MR2355596 (2008g:46081)

15. C. Jiang and R. Shi, Direct integrals of strongly irreducible operators. J. Ramanujan Math. Soc. 26 (2) (2011), 165-180. MR2816786

16. C. Jiang and Z. Wang, The spectral picture and the closure of the similarity orbit of strongly irreducible operators. Integral Equations Operator Theory 24 (1) (1996), 81-105. MR.1366542 (97h:47011)

17. C. Jiang and Z. Wang, Structure of Hilbert space operators. World Scientific Publishing Co. Pte. Ltd., Hackensack, NJ, 2006. MR2221863 (2008j:47001)

18. Z. Jiang and S. Sun, On completely irreducible operators. Front. Math. China 1 (4) (2006), 569-581. MR2257195 (2007g:47003)

19. H. Radjavi and P. Rosenthal, Invariant subspaces. Ergebnisse der Mathematik und ihrer Grenzgebiete, Band 77. Springer-Verlag, New York-Heidelberg, 1973. MR0367682 (51:3924)

20. J. T. Schwartz, $W^{*}$-algebras. Gordon and Breach, New York, 1967. MR0232221 (38:547)

School of Mathematical Sciences, Dalian University of Technology, Dalian, 116024, People's Republic of China

E-mail address: littlestoneshg@gmail.com 